\title{
APPLICATION OF RCM PRINCIPLES IN THE AIR OPERATIONS
}

Maintenance organizations assist in ensuring that all aircraft, their systems and parts are operated or maintained under controlled conformity to national and international airworthiness standards. Ensuring that these standards are met involves a vast network of organizations and personnel. To develop and then to comply with effective standards and procedures for maintenance program is never-ending process, which is responsible for the coordination and management of all maintenance activities and consequently manufacturing activities too. The RCM program provides a logical way of determining if preventive maintenance makes sense for a given part, system or the whole aircraft. The RCM concept completely changes the way in which the preventive maintenance is viewed. It is widely accepted fact that not all parts benefit from preventive maintenance.

\section{The Background}

Within the final twenty years of the last century, aircraft maintenance procedures have changed in general, perhaps more than any other management and technological discipline. The changes are due to a huge increase in the number and variety of physical assets (structural systems, equipment), which must be maintained on any place in the world, much more complex designs, new maintenance technologies, and changing policy of maintenance organization and its responsibilities.

Maintenance practices effectively respond to changing expectations. These include a rapidly growing awareness of the extent to which equipment failure affects safety and the environment, a growing awareness of the connection between maintenance and product quality, and increasing pressure to achieve high unit availability and to contain costs.

The changes are testing attitudes and skills in all branches of industry to the limit. Namely in aviation the maintenance people have to adopt completely new ways of thinking and acting, as engineers and as managers. At the same time the limitations of maintenance systems are becoming increasingly apparent, no matter how much they are computerized.

\section{Reliability Quantification}

A reliability-centred maintenance ( $\mathbf{R C M})$ program consists of a set of scheduled tasks generated on the basis of specific reliability characteristics of the aircraft they are designed to protect. The aircraft is composed of a number of systems and subsystems, a vast number of parts and assemblies. All these items can be expected to fail at one time or another, but some of the failures have more serious consequences than others. Certain kinds of failures have a direct effect on operating safety, and others affect the operational capability of the equipment. Operational reliability of an aircraft has to be defined and authoritatively prescribed with unique meaning anywhere adopted in the world.

The consequences of a particular failure depend on the design of the item and the equipment in which it is installed. One of the key goals of certification process and continued airworthiness standards in aviation is that each safety-critical structural part has a reliability of at least 0.999999999 ("nine 9's") per flight hour; in other words, the probability that a particular safety-critical system will fail is no more than one in a billion for each flight hour. Regulations seek to achieve this goal through a combination of requirements for design, analysis, test, inspection, maintenance, and operation. The development of rulemaking process in recent decades has been driven by the following factors:

- Increasing performance in air transportation with consequently increasing density of air traffic operations.

- Continued high level of public and administrative concerns in air transportation safety.

- The introduction of new technologies, which have advanced the efficiency of the air transportation system and provided opportunities to improve safe life of an aircraft.

- Lessons learned from investigations of civil aviation accidents and incidents.

- Changes in international air transportation regulations and policies.

\section{Reliability Centred Maintenance in Development Phase}

The envisaged operational conditions and real environment of aircraft operations form crucial factors for maintenance program development. The impact of failures on the aircraft airworthiness, and hence their consequences for the operating organization, are

\footnotetext{
* Luděk Beňo, Martin Bugaj, Andrej Novák

Faculty of Operation and Economics of Transport and Communication, Air Transport Departement,

E-mail: beno@fpedas.utc.sk, bugaj@fpedas.utc.sk, novak@fpedas.utc.sk
} 
predicted and established primarily by the aircraft designer. Experiences outgoing from recent similar design conception, predicted operational load cases with normal operational wear and the results from tests effectuated with simulated loadings on prototype can be used in this first step. On this level we are speaking about inherent reliability characteristics. failure could affect operating safety or have major economic consequences.

\section{Reliability Centred Maintenance in Operational Phase}

The definition of major economic consequences will vary from one operating organization to another, but in most cases it includes

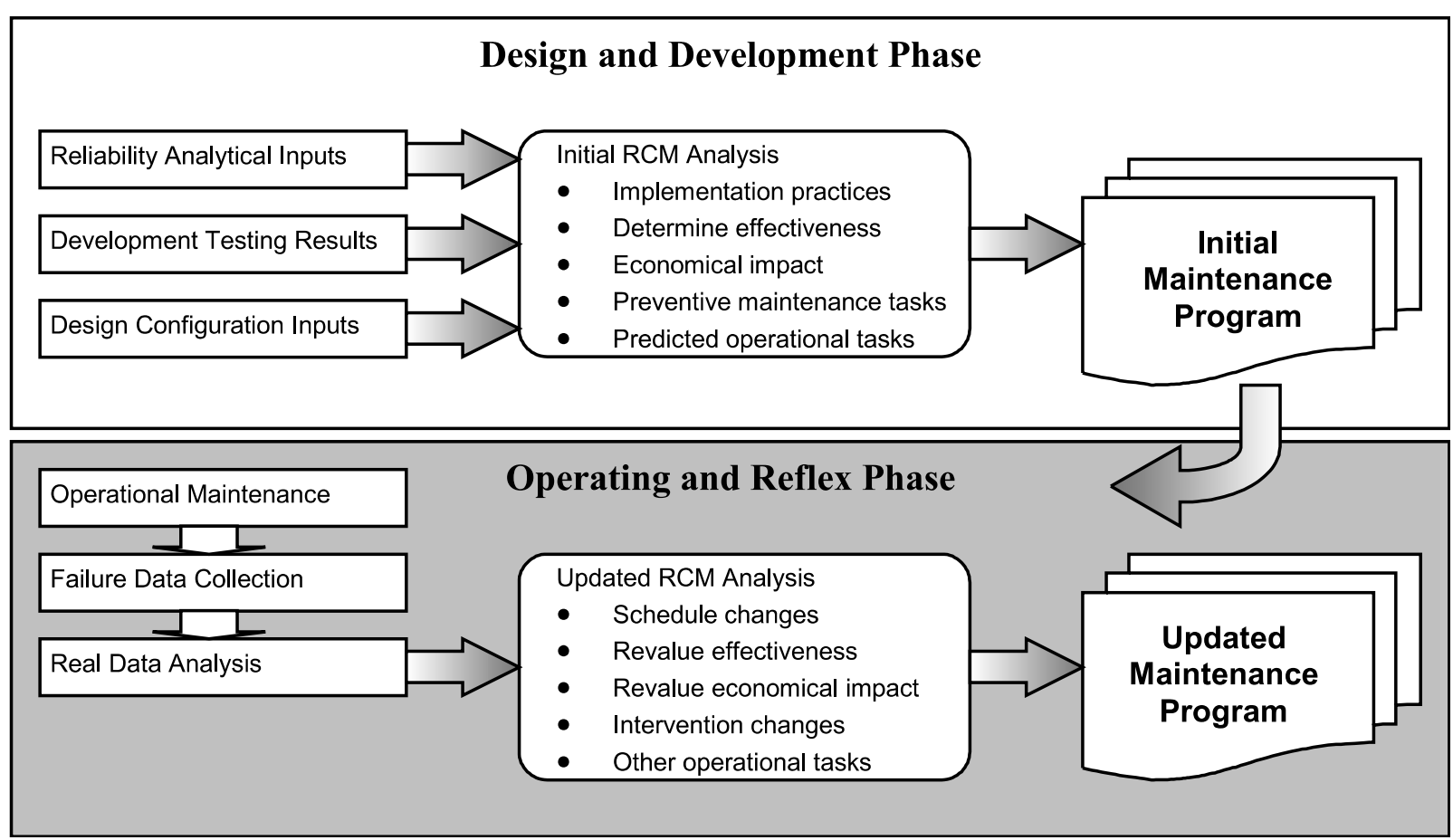

Fig. 1 How the RCM goes to real life

Expected impact on effectiveness and economics of aircraft operations have to be analysed with the key goal to make good and executable maintenance program. There are a great many items, of course, whose failure has no significance at the system or the whole aircraft level. These failures are tolerable, in the sense that the cost of preventive maintenance would outweigh the benefits to be derived from it. It is less expensive to leave these items in service until they fail than it is to try to prevent the failures. Most such failures are evident to the operating crew at the time they occur and are reported to the maintenance crew for corrective action.

Some items, however, have functions whose failure will not be evident to the operating crew. Although the loss of a hidden function has no direct consequences, any uncorrected failure exposes the aircraft to the consequences of a possible multiple failure as a result of some later second failure. For this reason items with hidden functions require special treatment in a scheduled maintenance program with preventive maintenance intervention.

The development of a maintenance program has to reduce the potential reliability problem by a quick, approximate, but conservative identification of a set of significant items - those items whose any failure that impairs the operational capability of the equipment or results in unusually high repair costs. At the same time all items with hidden functions must be identified, since they will be subjected to detailed analysis along with the significant items.

Once the aircraft is introduced into the service, the operational phase of maintenance program starts. The aircraft operator is obliged to develop credible system for collecting and reporting each accident and incident relevant to operational reliability. Structured database has to give responses helping in quantifying probability of repeated occurrences of registered events. Registered events relevant to operational reliability are failures, malfunctions or other types of faulty operation. The analysis itself is based on evaluation of the consequences for each type of malfunctions to which the item could be exposed. The logic used to organize this problem investigation and reporting, leads to categories of failure consequences:

- Safety consequences, which involve possible danger to the equipment and its occupants. Limits for random characteristics are standardised in aviation. 
- Operational consequences, which involve an indirect economic loss in addition to the cost of repair. Preventive maintenance actions have essential effect on operational costs in aviation.

- No operational consequences, which involve no economic loss other than the cost of repair applying RCM theory to aircraft. These failures have to be registered too, because potential future incident can be signalised.

\section{Operational Reliability Dataflow}

In the case of commercial aircraft continuous evolution of the design requirements promulgated by airworthiness authorities and the feedback of hardware information to designers by operating organizations have led to increasing capability for safe and reliable operation. Thus most modem aircraft enter service with design features for certain systems and items that allow easy identification of potential failures. Similarly, various parts of the airplane are designed for easy access when inspection is necessary or for easy removal and replacement of vulnerable items. A host of instruments and other indicators provide for monitoring of systems operation, and in nearly all cases essential functions are protected by some form of redundancy or by backup devices that reduce the consequences of failure to a less serious level.

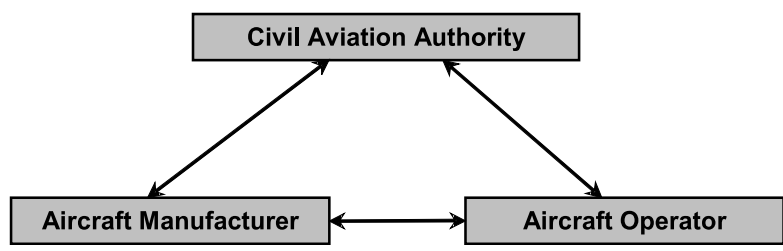

Fig. 2. Operational reliability data feedback

Complex equipment of the older generations of aircraft that has not benefited from such design practices will have different and less favourable reliability characteristics, therefore, less capability for reliable operation is envisaged. Since preventive maintenance is limited by the inherent characteristics of the equipment, in many cases RCM analysis can do little more than recommend the design changes that would make effective maintenance feasible.

The role of civil aviation authorities is to work with the operators and manufacturers of aircraft and engines to define and implement a proactive process that includes the following key elements:

- Data collection.

- Database management.

- Risk analysis.

- Risk management/action.

- Monitoring effectiveness.

Specific tasks should follow the process of RCM development:

- Manufacturers, with the advice and consent of operators and the appropriate civil aviation authority (CAA), should define data requirements and processes for sharing data. Comprehensive flight operations quality assurance systems should be used as a starting point.

- Operators should provide required data, as agreed upon.

- Manufacturers should solicit data from additional sources, such as International Civil Aviation Organization and others, to augment the operational database.

- Manufacturers, with oversight from the CAA and the assistance of operators, as required, should collect, organize, and analyze data to identify potential safety problems.

- Manufacturers should recommend corrective action for potential safety problems and seek consensus by operators. The CAA should make sure that actions proposed by manufacturers and operators will be effective, making regulatory changes and mandating compliance as appropriate.

- Manufacturers and operators, with oversight from the CAA, should monitor the effectiveness of corrective action and the safety management process.

The principles of reliability-centred maintenance still apply, and the decision questions are the same. The answers to these questions, however, must reflect the design characteristics of the aircraft.

\section{Summary of RCM Principles}

The complexity of modem aircraft generation makes it impossible to predict with any degree of accuracy when each part or each assembly is likely to fail. For this reason it is generally more productive to focus on those reliability characteristics that can be determined from the available information than to attempt to estimate failure behaviour that will not be known until the aircraft enters into the service. When developing an initial program, therefore, only a modest attempt is made to anticipate the operating reliability of every item. Instead, the governing factor in RCM analysis is the impact of a functional failure at the system level, and tasks are directed at a fairly small number of significant items - those whose failure might have safety or major economic consequences.

These items, along with all hidden-function items, are subjected to intensive study, first to classify them according to their failure consequences and then to determine whether there is some form of maintenance protection against these consequences.

The first step in this process is to organize the problem by partitioning the aircraft into object categories according to areas of engineering expertise. Within each of these areas the aircraft is further partitioned in decreasing order of complexity to identify significant items (those whose failure may have serious consequences for the aircraft as a whole), items with hidden functions (those whose failure will not be evident and might therefore go undetected), and non-significant items (those whose failure has no impact on operating capability). As this last group encompasses many thousands of items on an aircraft, this procedure focuses the problem of analysis on those items whose functions must be protected to ensure safe and reliable operation. 
The next step is a detailed analysis of the failure consequences in each case. Each function of the item under consideration is examined to determine whether its failure will be evident to the operating crew; if not, a scheduled-maintenance task is required to find and correct hidden failures. Each failure mode of the item is then examined to determine whether it has safety or other serious consequences. If safety is involved, scheduled maintenance is required to avoid the risk of a critical failure. If there is no direct threat to safety, but a second failure in a chain of events would have safety consequences, then the first failure must be corrected at once and therefore has operational consequences. In this case the consequences are economic, but they include the cost of operating capability lost as well as the cost of repair.

Whereas the criterion for task effectiveness depends on the failure consequences the task is intended to prevent, the applicability of each form of preventive maintenance depends on the failure characteristics of the item itself. For an on-condition task to be applicable there must be a definable potential failure condition and a reasonably predictable age interval between the point of potential failure and the point of functional failure. For a scheduled rework task to be applicable the reliability of the item must in fact be related to operating age; the age-reliability relationship must show an increase in the conditional probability of failure at some identifiable age (wear out) and most units of the item must survive to that age. The applicability of discard tasks also depends on the age reliability relationship, except that for safe life items the life limit is set at some fraction of the average age at failure. Failure finding tasks are applicable to all hidden function items not covered by other tasks.

\section{Development of RCM}

The process of developing an RCM program consists of determining which of these scheduled tasks, if any, are both applicable and effective for a given item. The fact that failure consequences govern the entire decision process makes it possible to use a structured decision diagram approach, both to establish maintenance requirements and to evaluate proposed tasks. The binary form of a decision diagram allows a clear focus of engineering judgment on each issue. It also provides the basic structure for a default strategy - the course of action to be taken if there is insufficient information to answer the question or if the study group is unable to reach a consensus. Thus if there is any uncertainty about whether a particular failure might have safety consequences, the default answer will be yes; similarly, if there is no basis for determining whether a proposed task will prove applicable, the answer, at least in an initial maintenance program, will be yes for on-condition tasks and no for rework tasks.

It is important to realize that the decision structure itself is specifically designed for the need to make decisions even with minimal information. For example, if the default strategy demands redesign and this is not feasible in the given timetable, then one alternative is to seek out more information in order to resolve the problem. However, this is the exception rather than the rule. In most cases the default path leads to no scheduled maintenance, and the correction, if any, comes naturally as real and applicable data come into being as a result of actual use of the aircraft in service.

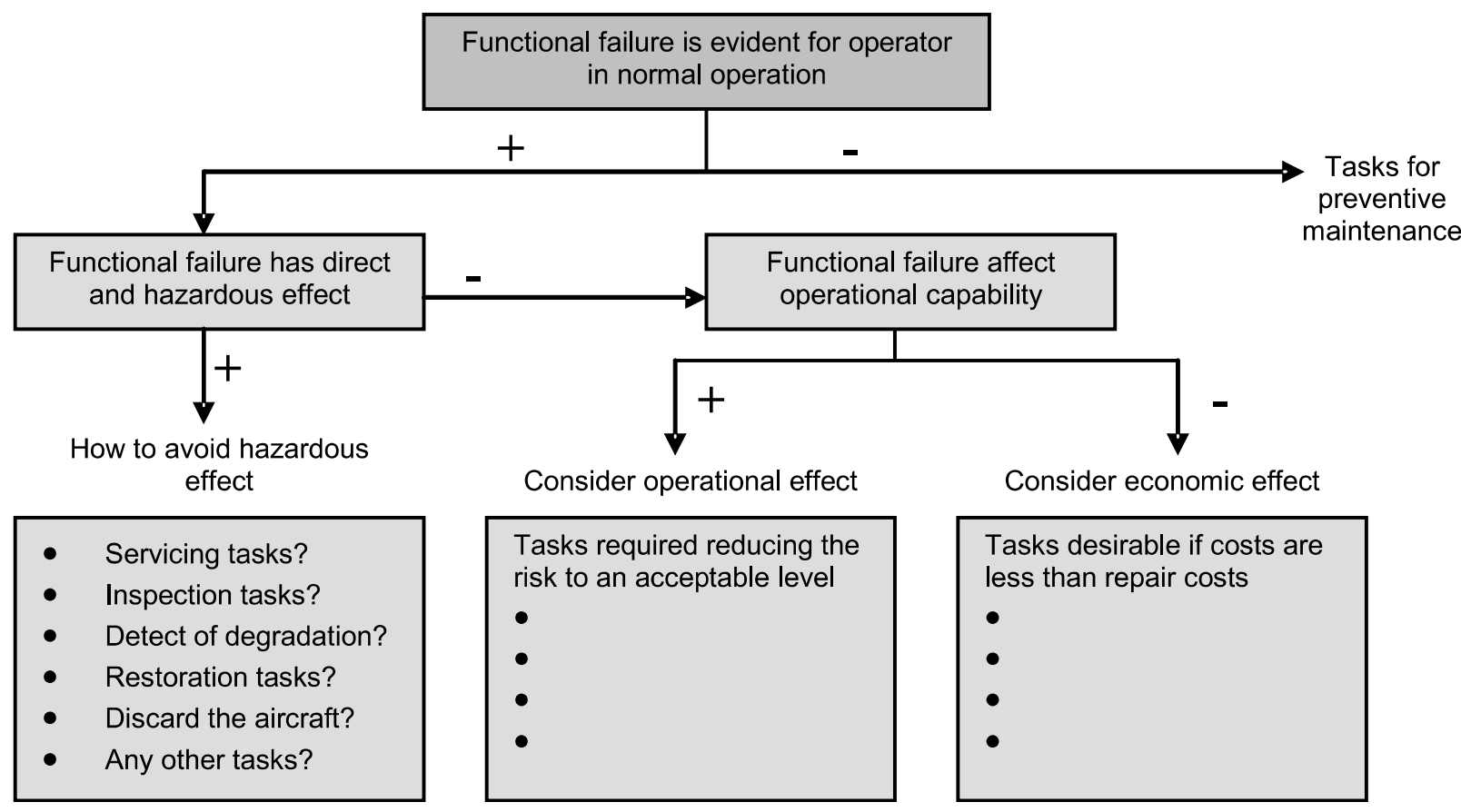

Fig. 3 Decision making 


\section{Decision Logic}

The decision logic also plays the important role of specifying its own information requirements. The first questions assure us that all failures will be detected and that any failures that might affect safety or operating capability will receive first priority. The remaining steps provide for the selection of all applicable and effective tasks, but only those tasks that meet the criteria are included. Again, real data from operating experience will provide the basis for adjusting default decisions made in the absence of information. Thus a prior-to-service program consists primarily of on-condition and sample inspections, failure finding inspections for hidden function items, and a few safe life discard tasks. As information is gathered to evaluate age reliability relationships and actual operating costs, rework and discard tasks are gradually added to the program where they are justified.
The net result of this careful bounding of the decision process is a scheduled maintenance program that is based at every stage on the known reliability characteristics of the aircraft in the operating context in which it is used. In short, reliability-centred maintenance is a well-tested answer to the paradox of modem aircraft maintenance - the problem of how to maintain the systems in a safe and economic fashion until we have accumulated enough information to know how to do it.

RCM will allow one to obtain the full design operating ability of the aircraft. It does not necessarily identify a new series of maintenance tasks. It identifies which tasks are most applicable, which are ineffective and provides a framework for developing an optimal preventive maintenance program.

\section{References}

[1] MOUBRAY, J.: Reliability-centred maintenance II, New York, Industrial Press inc., 2001

[2] SMITH, A.: Reliability-centred maintenance II, McGraw-Hill, 1992

[3] AUGUST. J.: Applied Reliability-centred maintenance II, Pennwell Corp., 2000

[4] KROES. M. J.: Aircraft maintenance and repair, McGraw-Hill College, 1999

[5] BUGAJ. M.: Aircraft Operational Technology - maintenance impact on aircraft reliability, University of Žilina, 2002 